\title{
Learning convex bodies under uniform distribution
}

\author{
W. Kern \\ Faculty of Applied Mathematics, Unicersity of Twente, P.O. Box 217, 7500 AE Enschede, The Netherlands \\ Communicated by T. Lengatue \\ Received 15 October 1991 \\ Revised 15 April 1992
}

Abstract

Kern, W., Learning convex bodies under uniform distribution. Information Processing Letters 43 (1992) 35-39.

We prove that the class of convex bodies contained in a fixed (prescribed) bounded region $R \subseteq \mathbb{R}^{d}$ is PAC-learnable, if the positive examples are drawn according to the uniform distribution $D^{+}$on the target concept (the distribution $D^{-}$on the negative examples may be arbitrary). Our results extend previous work by E. Baum.

Keywords: Theory of computation, learning by example, convex bodies, uniform distribution

\section{Introduction}

We study the problem of learning the class $\mathscr{C}_{R}$ of convex bodies contained in a fixed (prespecified) region $R \subseteq \mathbb{R}^{d}$. According to Valiant's $[7,8]$ general model, the learning problem can be described as follows. Given $K \in \mathscr{C}_{R}$, a distribution $D^{+}$on $K$ and a distribution $D^{-}$on $R \backslash K$ and $\varepsilon>0$, we say that $H \in \mathscr{C}_{R}$ is an $\varepsilon$-good approximation of $K$ with respect to $D^{+}$and $D^{-}$if

$\varepsilon^{+}:=D^{+}(K \backslash H) \leqslant \varepsilon$

and

$\varepsilon^{-}:=D^{-}(H \backslash K) \leqslant \varepsilon$.

Assume that for any $K \in \mathscr{C}_{R}$ and any $D^{+}$and $D^{-}$as above there is a black box with two buttons labeled "+" and "- ". Pushing the "+" button causes the black box to generate a random posi-

Correspondence to: W. Kern, Faculty of Applied Mathematics, University of Twente, P.O. Box 217, 7500 AE Enschede. The Netherlands. tive example, i.e. it generates a random point $x \in K$ with respect to the distribution $D^{+}$on $K$. Similarly, if we push the "-" button, we get a random negative example, i.e. a random point $x \in R \backslash K$, generated with respect to the distribution $D^{-}$on $R \backslash K$.

An algorithm $L$ is said to ( $P A C$-) learn the class $\mathscr{C}_{R}$ if, given any $\varepsilon>0$, any $\delta>0$, any hidden (unknown) target concept $K \in \mathscr{C}_{R}$ and any pair of (unknown) distributions $D^{+}, D^{-}$on $K$ resp. $R \backslash$ $K$, the algorithm $L$ computes with probability at least $1-\delta$ and $\varepsilon$-good approximation $H$ of $K$ with respect to $D^{+}$and $D^{-}$, using only a number of random examples generated by the black box associated to $K, D^{+}$and $D^{-}$. The computation time should be polynomial in $1 / \varepsilon$ and $1 / \delta$. Generation of a (positive or negative) example counts as a single step. Hence, in particular the number of examples generated during the computation must be polynomial in $1 / \varepsilon$ and $1 / \delta$. (We consider an example $x$ as a real vector in $\mathbb{R}^{d}$. A more detailed analysis would have to take the size of $x$ into account.) 
It is easy to see that the class of convex bodies even in the 2-dimensional case has unbounded VC-dimension and hence cannot be learned in the above sense, cf. e.g. [5]. In this paper by Long and Warmuth, one can find also a lot of related problems concerning learnability (or "predictability") of geometric concepts and more references. For example, they study the problem of learning vertex represented polytopes. Baum in [2] deals with learning unions of $k$ halfspaces.

A natural question that comes up, as soon as one notices that a class $\mathscr{E}$ is not learnable in the sense described above, is: what happens if we consider the fixed distribution analogue, i.e. the case where $D^{+}$and $D^{-}$are known in advance? In Section 2 we will show that if $D^{+}$is (known to be) the uniform distribution, then $\mathscr{C}_{R}$ becomes learnable, i.e. there exists an algorithm which, for any $\varepsilon>0, \delta>0, K \in \mathscr{C}_{R}$, and any distribution $D^{-}$on $R \backslash K$, finds a hypothesis $H \in \mathscr{C}_{R}$ such that

$\varepsilon^{+}:=D^{+}(K \backslash H)=\frac{\operatorname{vol}(K \backslash H)}{\operatorname{vol}(K)} \leqslant \varepsilon$

and

$\varepsilon^{-}:=D^{-}\left(H \backslash K^{*}\right) \leqslant \varepsilon$.

The algorithm is the most obvious one: We first generate $n$ random positive examples $x \in K$ and then let the hypothesis $H$ consist of their convex hull. Thus we always have $H \subseteq K$ and hence $\varepsilon^{-}=0$, whatever the distribution $D^{-}$may be. The crucial point is to show that the sample size $n$ may be chosen to be polynomial in $1 / \varepsilon$ and $1 / \delta$ in order to ensure that $\varepsilon^{+} \leqslant \varepsilon$ with probability at least $1-\delta$.

Most closely related to our investigation is a result by Baum [3] on learning the class $\mathscr{C}_{Q}$ of convex sets contained in the unit cube $Q \subseteq \mathbb{R}^{a}$. In contrast to our work, however, Baum considers the problem where examples (both positive and negative ones) are drawn from the uniform distribution $D$ on $Q$. Hence, in his setting, a hypothesis $H \in \mathscr{C}_{Q}$ is an $\varepsilon$-good approximation for $K \in$ $\mathscr{C}_{Q}$, if

$$
\frac{\operatorname{vol}(H \Delta K)}{\operatorname{vol}(Q)}=\operatorname{vol}(H \Delta K) \leqslant \varepsilon
$$

This looks quite similar to our setting, and even the algorithm proposed in [3] is the same as ours. However, the analysis presented there cannot be applied in a straightforward way to our problem.

The reason is that, in Baum's model any concept $K$ with $\operatorname{vol}(K) \leqslant \varepsilon$ may feasibly approximated by hypothesis $H:=\emptyset$. As will become clear in Section 2, this is precisely the point where problems arise: We will have to adapt Baum's analysis to the case where $K$ is not "well rounded" i.e. "ton flat", and hence has negligible volume.

\section{The main result}

In this section, we will show that it is possible to learn the class of convex bodies contained in a prespecified bounded region $R \subseteq \mathbb{R}^{d}$, if the distribution $D^{+}$on the positive examples is uniform.

We start by presenting some simple facts about convex bodies; cf. also [4].

Lemma 2.1. Let $K \subseteq \mathbb{R}^{d}$ be a convex body containing the unit ball $B \subseteq \mathbb{R}^{d}$ and let $\alpha \geqslant 0$. Then any $y \in \mathbb{R}^{d} \backslash(1+\alpha) K$ has distance at least $\alpha$ from $K$.

Proof. Let $y \in \mathbb{R}^{d} \backslash(1+\alpha) K$. Choose an inequality $v \cdot x \leqslant 1+\alpha$ separating $y$ from $(1+\alpha) K$. Thus, $v \cdot y>1+\alpha$ and $v \cdot(1+\alpha) z \leqslant 1+\alpha$ for any $z \in K$. Thus, if $z \in K$, we get $v \cdot z \leqslant 1$, hence $v \cdot(y-z)>\alpha$. Now recall that the unit ball $B \subseteq$ $K$. Hence in particular $v \cdot z \leqslant 1$ for $z=v /\|v\| \in$ $B$, which implies $\|v\| \leqslant 1$. Thus we conclude that $\|y-z\| \geqslant\|y-z\| \cdot\|v\| \geqslant\left|\left(\begin{array}{ll}y & z\end{array}\right) \cdot v\right|>\alpha$.

Lemma 2.2. Let $K \subseteq \mathbb{R}^{d}$ be a convex body containing the unit ball $B \subseteq \mathbb{R}^{d}$ and let $\alpha \leqslant 1$. If $y \in(1-$ $\alpha) K$, then $y$ has distance at least $\alpha$ from the boundary of $K$.

Proof. Let $y \in(1-\alpha) K$ and $z \in \partial K$. Then there exists a vector $v$ such that $v \cdot z=1$ and $v \cdot x \leqslant 1$ for all $x \in K$. Thus, $v \cdot y \leqslant 1-\alpha$, implying that $v(z-y) \geqslant \alpha$. Again, $B \subseteq K$ shows that $\|v\| \leqslant 1$, hence $\|z-y\| \geqslant \alpha$. 
Now consider a lattice $\sigma \mathbb{Z}^{d} \subseteq \mathbb{R}^{d}$ and the corresponding dissection of $\mathbb{R}^{d}$ into cubes of side length $\sigma$. We will assume that $\sigma \leqslant 1 / 2 d^{3 / 2}$. Say that two cubes are adjacent, if they have at least one point in common (hence, every cube is adjacent to $3^{d}-1$ other cubes). The set of cubes adjacent to a cube $Q$ is called the neighborhood of $Q$.

Given a convex body $K \subseteq \mathbb{R}^{d}$, let $K^{I}$ be the set of cubes whose neighborhood is completely contained in $K$. Let $K^{B}$ denote the set of cubes whose neighborhood intersects $K$, but is not completely contained in $K$.

Lemma 2.3. If $K$ is a convex body containing the unit ball and $\sigma \leqslant 1 / 2 d^{3 / 2}$, then

$$
\frac{\operatorname{vol}\left(K^{B}\right)}{\operatorname{vol}(K)} \leqslant 8 \sigma d^{3 / 2} \text {. }
$$

Proof. Observe that the distance between any point in $\partial K$ and any point in $K^{B}$ is at most $2 \sigma \sqrt{d}$, since this is twice the diameter of a cube of side-length $\sigma$. From the two previous lemmata we conclude that

$$
(1-2 \sigma \sqrt{d}) K \subseteq K^{B} \subseteq(1+2 \sigma \sqrt{d}) K .
$$

Using $(1+x / d)^{d} \leqslant \mathrm{e}^{x} \leqslant 1+3 x$ and $(1 \quad x / d)^{d} \geqslant$ $1-x$ for $0 \leqslant x \leqslant 1$, we get

$$
\begin{aligned}
\operatorname{vol}\left(K^{B}\right) & \leqslant\left((1+2 \sigma \sqrt{d})^{d}-(1-2 \sigma \sqrt{d})^{d}\right) \operatorname{vol}(K) \\
& \leqslant 8 \sigma d^{3 / 2} \operatorname{vol}(K) .
\end{aligned}
$$

Now let us turn to learnability of convex bodies. Our first result concerns learnability of "well rounded" convex bodies.

Definition. A convex body $K \subseteq \mathbb{R}^{d}$ is well rounded, if

$B \subseteq K \subseteq d \cdot B$.

Proposition 2.4. Let $R \subseteq \mathbb{R}^{d}$ be a bounded region containing the unit ball. Then the class of wellrounded convex bodies $K \subseteq R$ is learnable, if the distribution $D^{+}$on the positive examples is uniform.
Proof. Let $K \subseteq R$ be the concept to be learned and let $\delta$ and $\varepsilon$ be the security and error parameters, resp. Our learning algorithm is the obvious one: We first pick a sample of positive examples $x^{(1)}, \ldots, x^{(n)} \in K$. (The sample size $n$, necessary to achieve the desired accuracy bound $\varepsilon$ will be determined below.) We then take as hypothesis $H$ the convex hull of these positive examples. Thus, our hypothesis $H$ will always be a subset of the hidden target concept $K$ and therefore we will only have to bound the term

$\varepsilon^{+}:=\frac{\operatorname{vol}(K \backslash H)}{\operatorname{vol}(K)}$,

since $\varepsilon^{-}$is always equal to 0 .

Let $\sigma:=\varepsilon / 8 d^{3 / 2}$ and consider the lattice $\sigma \mathbb{Z}^{d}$. By our choice of $\sigma$ and Lemma 2.3, the number $N$ of cubes in $K^{I} \cup K^{B}$ is bounded by

$\frac{\operatorname{vol}(K)}{\sigma^{d}} \leqslant N \leqslant(1+\varepsilon) \frac{\operatorname{vol}(K)}{\sigma^{d}}$.

The probability of missing a cube $Q$ fully contained in $K$ when drawing a random point from $K$ is given by

$p=1-\frac{\sigma^{d}}{\operatorname{vol}(K)} \leqslant 1-\frac{1}{N}$.

Thus, drawing a sample of size $n=N \cdot \log (N / \delta)$, the probability of missing any one of these cubes is bounded by

$N \cdot\left(1-\frac{1}{N}\right)^{n} \leqslant \delta$

Note that this sample size is feasible, since, due to the well-roundedness of $K$, we have $\operatorname{vol}(K)=$ $\mathrm{O}\left(d^{d}\right)$, which is constant, since $d$ is fixed.

Our learning algorithm takes as hypothesis $H$ the convex hull of those sample points which are in $K$. Since, with probability at least $1-\delta$, every cube fully contained in $K$ is hit at least once, we have

$H \supseteq \bigcup K^{I}$ with probability $\geqslant 1-\delta$. 


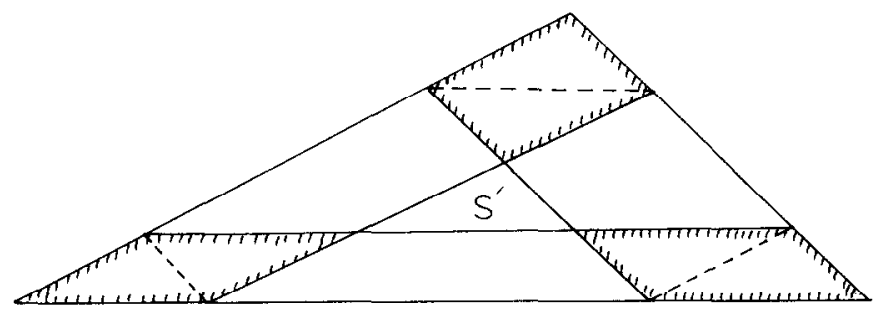

Fig. 1.

Hence, by Lemma 2.3 , the error is bounded by

$$
\begin{aligned}
\frac{\operatorname{vol}(K)-\operatorname{vol}(H)}{\operatorname{vol}(K)} & \leqslant \frac{\operatorname{vol}\left(K^{B}\right)}{\operatorname{vol}(K)} \\
& \leqslant 8 \sigma d^{3 / 2} \leqslant \varepsilon .
\end{aligned}
$$

Now assume that $R \subseteq \mathbb{B}^{d}$ is any fixed bounded region and we want to learn arbitrary convex sets $K \subseteq R$. The key result we use is the following well-known fact, due to Löwner:

Theorem 2.5 [6]. If $K \subseteq \mathbb{R}^{d}$ is a bounded closed convex set, then there exists an affine transformation $f: \mathbb{R}^{d} \rightarrow \mathbb{R}^{d}$ such that $f(K)$ is well rounded.

In other words, given $K \subseteq R$, there exists a lattice $\Gamma=f^{-1}\left(\sigma \mathbb{Z}^{d}\right)$, such that $K$ with respect to $\Gamma$ behaves like a well-rounded body with respect to $\sigma \mathbb{Z}^{d}$. More precisely, if we define the sets $K^{l}$ and $K^{B}$ of parallelepipeds in $\Gamma$ in the obvious way, then an anologue of Lemma 2.3 follows and the proof of Proposition 2.4 "transforms" into a proof for the following:

Theorem 2.6. Let $R \subseteq \mathbb{R}^{d}$ be a fixed bounded region. Then the class of convex bodies $K \subseteq R$ is learnable, if the distribution $D^{+}$on the positive examples is uniform.

Proof (sketch). Note that affine transformations preserve convex hulls, uniform distributions and relative volumes.

As a matter of curiosity, we would like to remark that it is also possible to "learn" (an approximation of) the mapping $f$, which well- rounds a hidden target concept $K \subseteq R$. For example, from Löwner's theorem one immediately gets that there exists a simplex $S$ such that

$S \subseteq K \subseteq d^{2} * S$,

where "*" denotes "blow up". If we consider the slightly smaller simplex $S^{\prime}:=\frac{1}{4} * S$, then it is not difficult to see that there is a significant chance of picking $d+1$ random points in $S$ whose convex hull contains $S^{\prime}$. (Figure 1 indicates that this chance is at least $\left[2 \cdot \operatorname{vol}\left(S^{\prime}\right) / \operatorname{vol}(K)\right]^{d+1} \geqslant$ $\left(1 / 4 d^{2}\right)^{d}$.)

Hence, we may proceed as follows. In each stage we start from scratch by drawing $d+1$ points in $K$. We let $S^{\prime}$ denote their convex hull. We then check whether $S:=4 d^{2} S^{\prime}$ contains (almost all of) $K$ by again drawing a sample of appropriate size from $K$. With high probability, we will succeed within a polynomial number of stages.

Note that the above (sketchy) argument offers an alternative proof for Theorem 2.6: Once we have found a simplex $S^{\prime} \subseteq K$ as described above, we may apply the transformation $f$ which sends $S^{\prime}$ to a regular simplex containing the unit ball and then "learn" the transformed convex body $f(K)$ as described in the proof of Theorem 2.5 . (Note that $f(K)$ is "almost" well rounded in the obvious sense.)

\section{Remarks}

We described an algorithm for learning the class $\mathscr{C}_{R}$ of convex bodies contained in a fixed bounded region $R \subseteq \mathbb{R}^{d}$ under uniform distribution on the positive examples. The sample size 
(and hence, the running time) used by the algorithm is exponential in $d$. The following result, due to Barany and Füredi, seems to indicate that this is best possible:

Theorem 3.1 [1]. Let $V(n, d)$ denote the maximum volume of the convex hull of $n$ points from the unit ball $B^{d} \subseteq \mathbb{R}^{d}$. If $n=d^{a}$, then, for sufficiently large $d$,

$\frac{V(d, n)}{\operatorname{vol}\left(B^{d}\right)} \leqslant\left(\frac{2 a \log d}{d}\right)^{d / 2}$.

This shows that our simple learning algorithm (taking the convex hull of the positive examples as hypothesis) cannot succeed unless

$a \approx d / \log d$,

i.e. the sample size is $n=d^{a} \approx 2^{d}$. This does not prove, of course, that any learning algorthm needs an exponential number of examples. A particularly interesting question arises when $R=B^{d}$ is the unit ball and both $D^{+}$and $D^{-}$are known to be uniform.

\section{References}

[1] 1. Barany and 7. Füredi, Computing the volume is difficult, Discrete Comput. Geom. 2 (1987) 319-326.

[2] E. Baum, On learning a union of halfspaces, $J$. Complexity 6 (1990) 67-101.

[3] E. Baum, The perceptron algorithm is fast for nonmalicious distributions, Neural Computat. 2 (1990) 248-260.

[4] M. Dyer, A. Frieze and R. Kannan, A random polynomial time algorithm for estimating volumes of convex bodies, Preprint, Carnegie Mellon University, 1988.

[5] P.M. Long and M.K. Warmuth, Composite geometric concepts and polynomial predictability, in: Proc. 3rd Ann. Workshop on Computational Learning Theory (1990) 273287.

[6] L. Lovasz, An algorithmic theory of numbers, graphs and convexity, Preprint, Bonn University, 1985.

[7] L. Valiant, A theory of the learnable, Comm. ACM 27 (11) (1984).

[8] L. Valiant, A view of computational learning thcory, Working paper, Harvard University, 1990. 